\title{
Beauty Production at HERA
}

on behalf of the ZEUS and $\mathrm{H} 1$ Collaborations
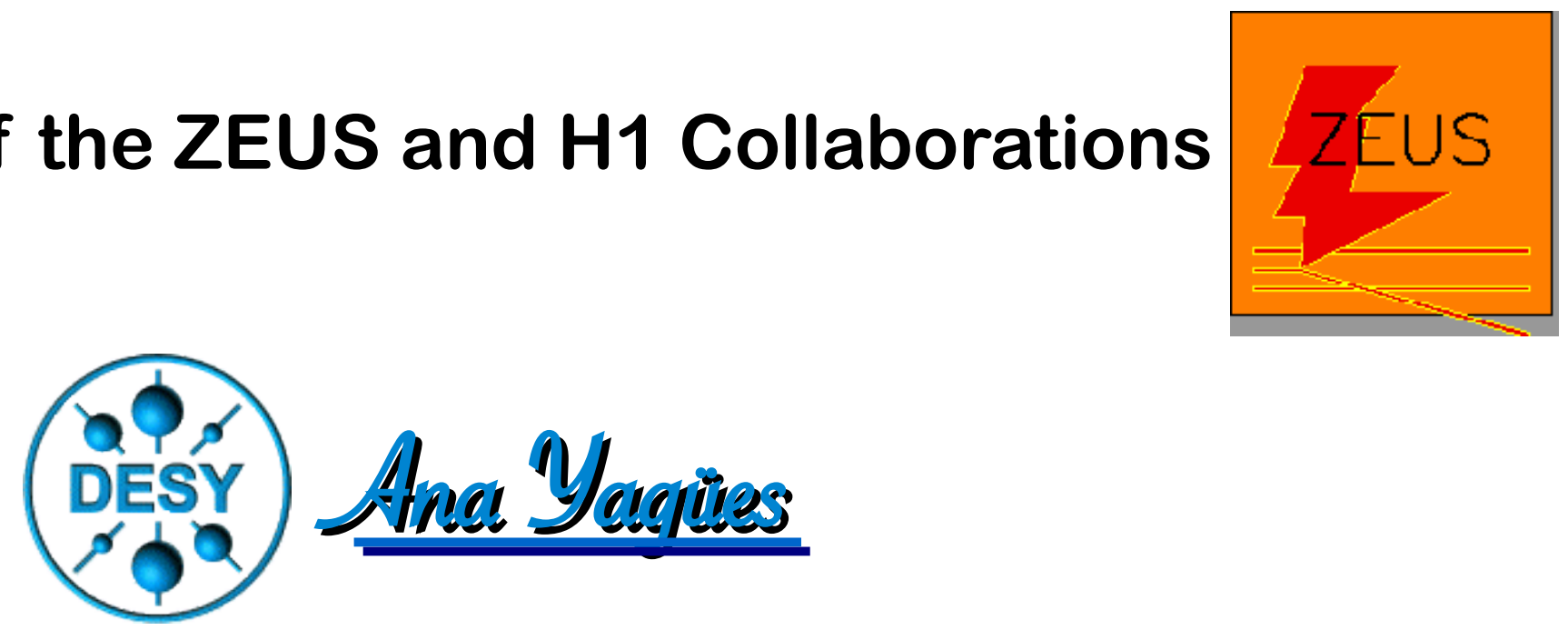

10"' COMFERENCE ON THE INTERSECTIONS OF PARTTCLE AMD NUCLEAR PHYSSICS San Diego, California-26 May to 31 May, 2009 


\section{Outline}

$\checkmark$ HERA Collider and ZEUS/H1 detectors

$\checkmark$ Heavy flavour production at HERA

$\checkmark$ Latest beauty results at HERA

(Photoproduction PHP, $\mathrm{Q}^{2} \approx 0$, and Deep Inelastic Scattering DIS, $\mathrm{Q}^{2} \geqslant 1 \mathrm{GeV}^{2}$ )

PHP events with 2 jets + sec.vtx (ZEUS-prel-09-005) PHP events with 2 jets $+1 \mu$ (DESY-08-210, by ZEUS Coll.) DIS decays into $\mu$ 's (DESY-09-56 by ZEUS Coll.) DIS events using sec.vtx (H1-prel-08-173 by H1 Coll.) 


\section{The HERA Collider}
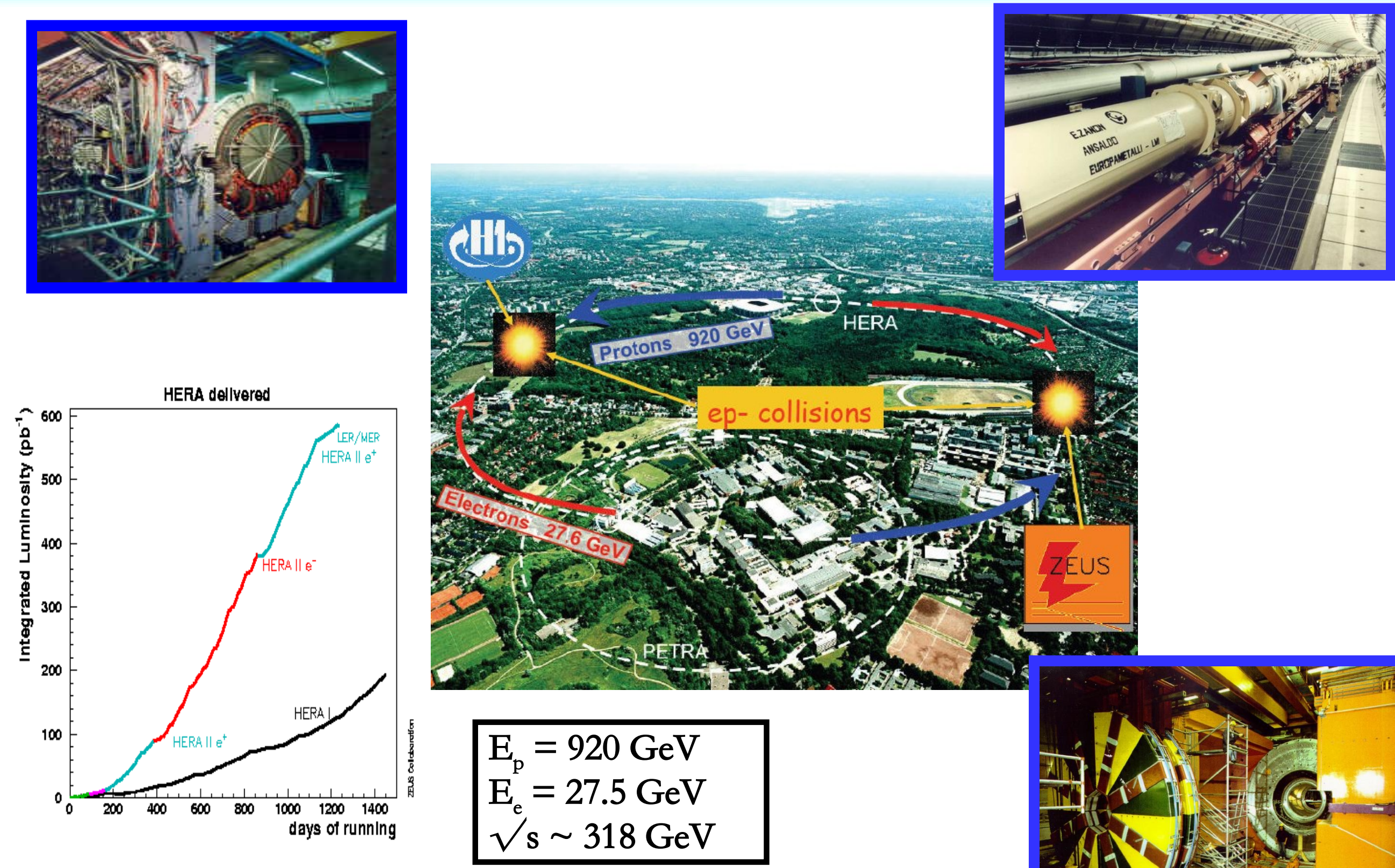

Ana Yagües

CIPANP 2009

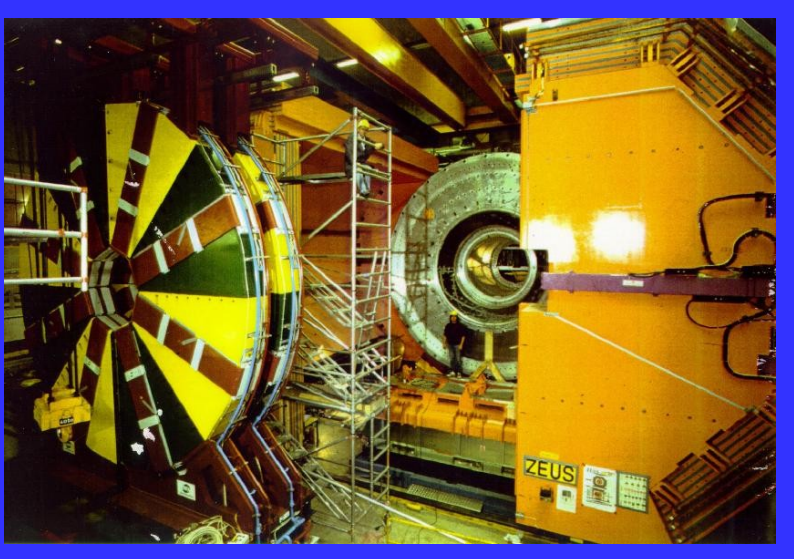




\section{Heavy Flavour Production}

\section{Dominant production process in ep-collisions: Boson-Gluon Fusion}

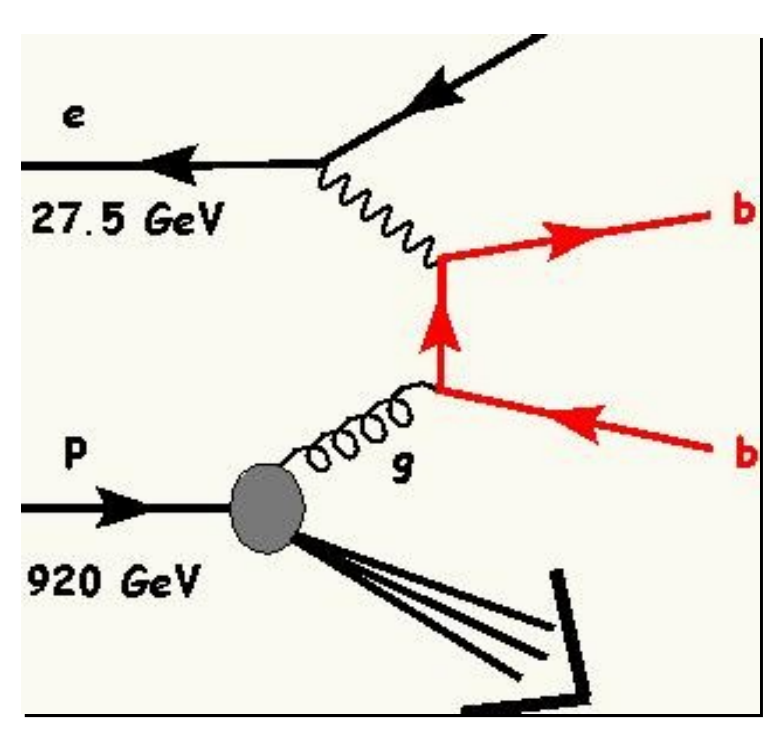

Why study heavy quark production at HERA?:

Test of perturbative QCD due to the hard scale given by the heavy quark mass.

Study of multi-scale problem: $\mathrm{p}_{\mathrm{T}}, \mathrm{m}^{2}, \mathrm{Q}^{2}$

* Better understanding of structure of the proton: direct sensitive to gluon content inside proton (PDF's). beauty measurements at high $\mathrm{Q}^{2}$ relevant for $\mathrm{LHC}$ e.g. $\mathrm{bb} \rightarrow \mathrm{H}$

\section{Kinematic Variables:}

$\mathrm{Q}^{2}=-\mathrm{q}^{2}=\left(\mathrm{k}-\mathrm{k}^{\prime}\right)^{2} \quad \frac{\text { Neg. squared momentum transfer }}{\text { (virtuality of exchanged boson) }}$

$\mathrm{s}=(\mathrm{k}+\mathrm{p})^{2} \approx 4 \mathrm{E}_{\mathrm{e}} \mathrm{E}_{\mathrm{p}} \quad$ CM Energy, at HERA $\sqrt{ } \mathrm{s}=318 \mathrm{GeV}$

$x=Q^{2} / 2 p \bullet q \quad$ Biorken scaling variable: momentum fraction of parton interacting with lepton in infinite momentum frame (QPM)

$y=\mathrm{p}^{\bullet} \mathrm{q} / \mathrm{p} \bullet \mathrm{k} \quad$ Inelasticity: lepton momentum fraction transferred to boson in proton rest frame 


\section{pQCD approximations \\ (assuming one dominant hard scale)}

Massive scheme/FO: $\rightarrow \mathrm{m}_{\mathrm{b}}$

(Fixed Flavour Number Scheme)

- b massive

- neglects $\left[\alpha_{\mathrm{s}} \ln \left(\mathrm{Q}^{2} / \mathrm{m}_{\mathrm{b}}{ }^{2}\right)\right]^{\mathrm{n}} \ldots$

- reliable for $\mathrm{p}_{\mathrm{T}} \sim \mathrm{m}_{\mathrm{Q}}$

- FMNR (PHP) and HVQDIS (DIS)

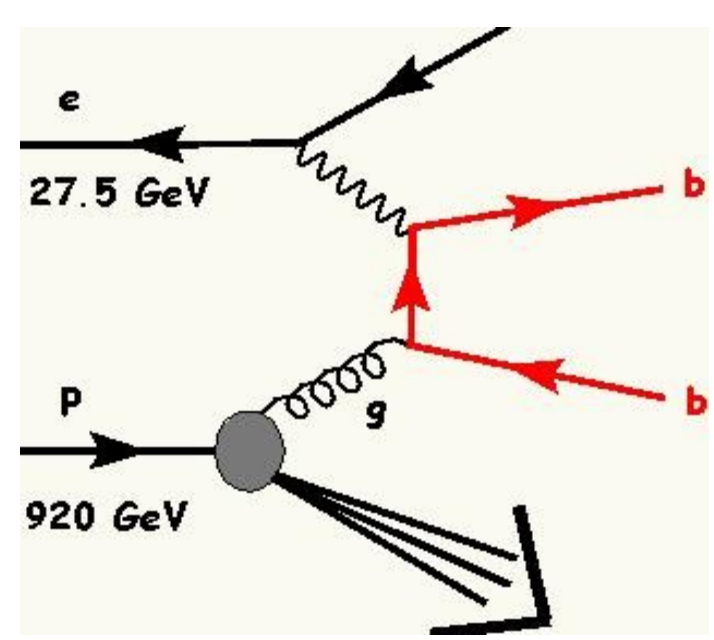

Massless scheme/NLL:

$\rightarrow \mathrm{Q}^{2}, \mathrm{p}_{\mathrm{T}}$

- b massless

- resums $\left[\alpha_{\mathrm{s}} \ln \left(\mathrm{Q}^{2} / \mathrm{m}_{\mathrm{b}}{ }^{2}\right)\right]^{\mathrm{n}} \ldots$

- reliable for $\mathrm{p}_{\mathrm{T}}>>\mathrm{m}_{\mathrm{Q}}$
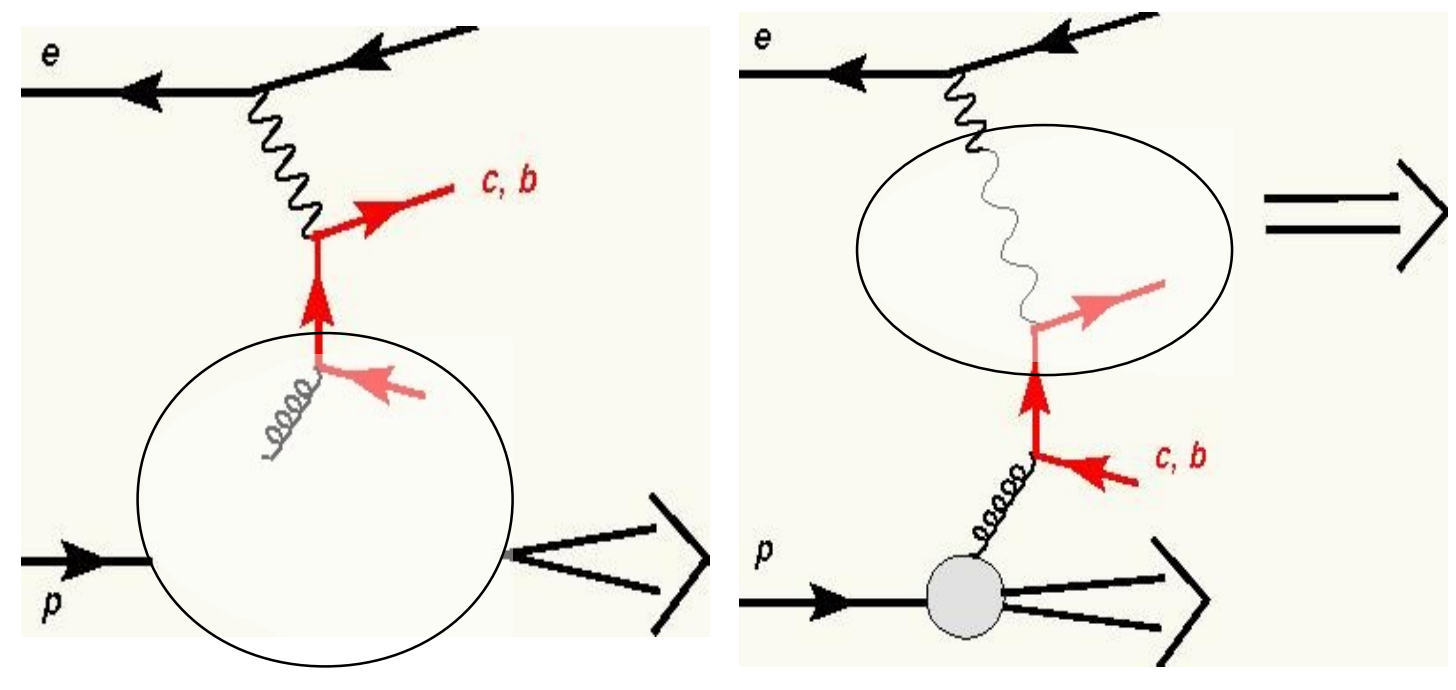

Variable schemes:

(Variable Flavour Number Scheme)

- at small $\mathrm{Q}^{2} \rightarrow$ massive, at large $\mathrm{Q}^{2} \rightarrow$ massless

- MRST, CTEQ... 


\section{Beauty PHP from inc. secondary vertexing}

The experimental method and data selection

$\mathcal{L}=128 \mathrm{pb}^{-1}\left(06 / 07 \mathrm{e}^{ \pm} \mathrm{p}\right.$ data $)$

PHP selection:

veto on scattered $\mathrm{e}^{ \pm}$

$0.2<\mathrm{y}_{\mathrm{JB}}<0.8$

JET selection:

2 Jets (kt-algorithm, massive) with $\mathrm{p}_{\mathrm{T}}>7(6) \mathrm{GeV}$ and $|\eta|<2.5$

TRACK selection: $\mathrm{p}_{\mathrm{T}}>0.5 \mathrm{GeV}$

_If $\geqslant 2$ tracks associated to a jet $\rightarrow$ secondary vertex fitted $-1.6<\eta_{\text {jet }}<1.3$ for vtx-tagged jet

Ana Yagües

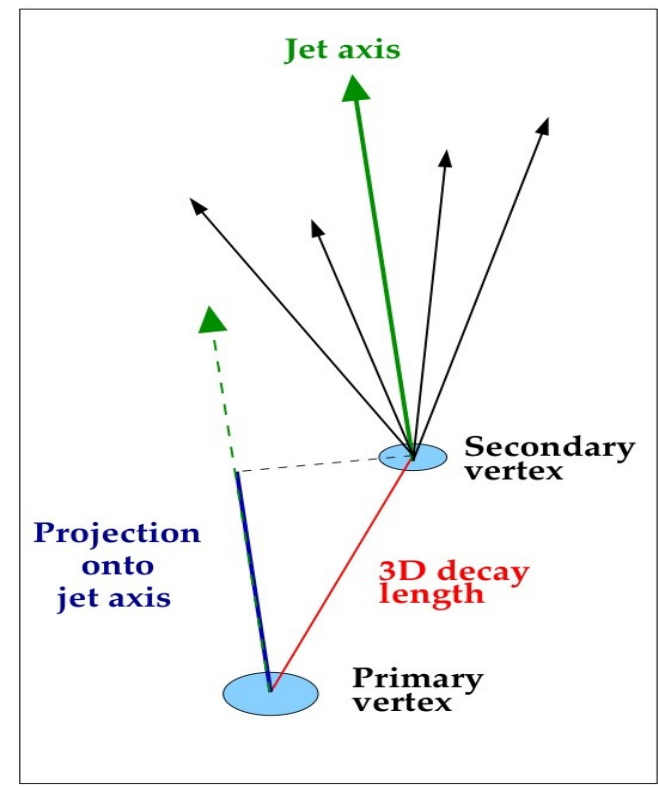

Combining decay length significance $\left(\mathrm{S}=\mathrm{L}_{\mathrm{xy}} / \sigma\right)$ and mass of sec.vtx $\left(\mathrm{m}_{\mathrm{vtx}}\right)$ provides very good discrimination between beauty and BG:

$\rightarrow \mathrm{S}$ divided in 3 mass bins
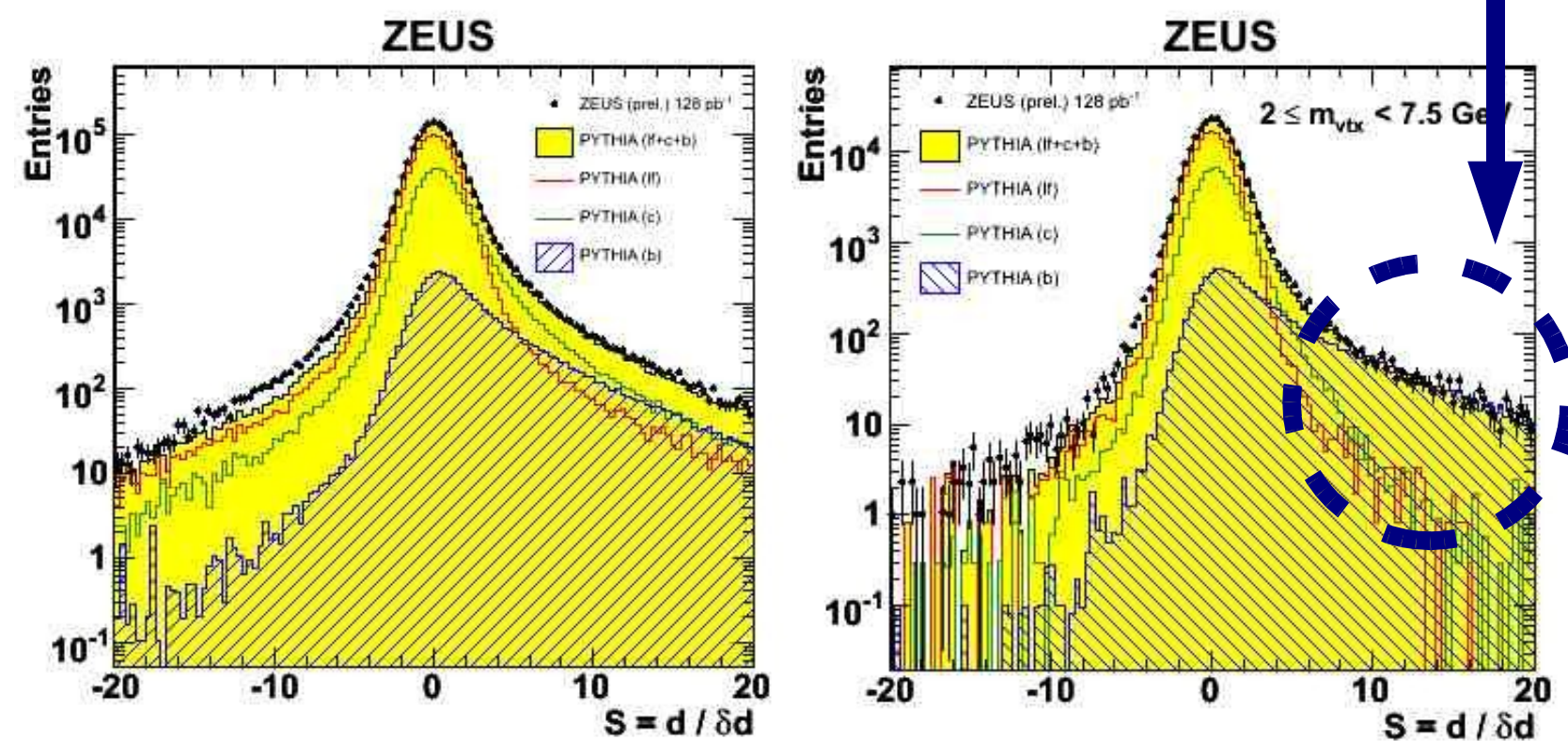


\section{Beauty PHP from inc. secondary vertexing (cont'd)}

Control distributions of beauty-einriched region
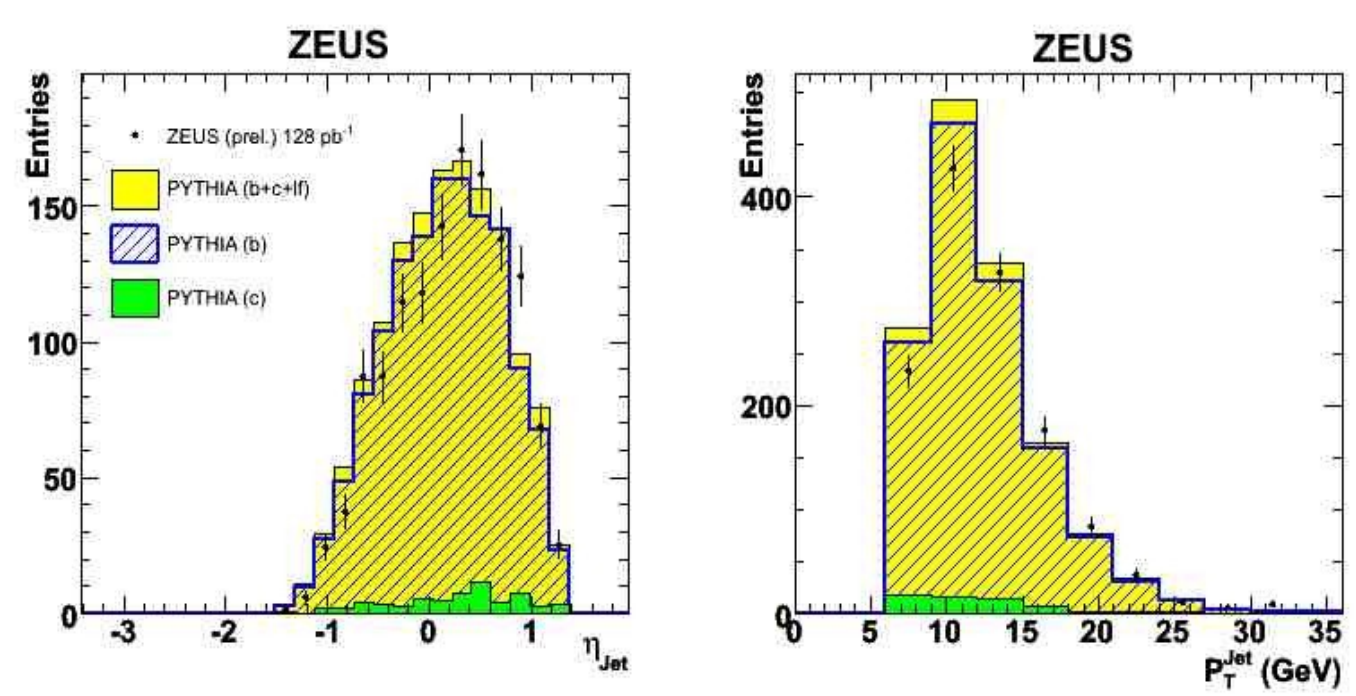

\section{The signal extraction}

To reduce systematic uncertainties:

$\rightarrow$ mirrored distributions ( $\left.\mathrm{S}^{+}-\mathrm{S}^{-}\right)$

Fractions of $b, c$ and If extracted using $\chi^{2}$-simultaneous fit to the 3 mass bins mirrored distributions

- Almost pure beauty sample: $>\mathrm{m}_{\mathrm{vtx}}>2 \mathrm{GeV}$ and $\left(\mathrm{S}^{+}-\mathrm{S}^{-}\right)>8$

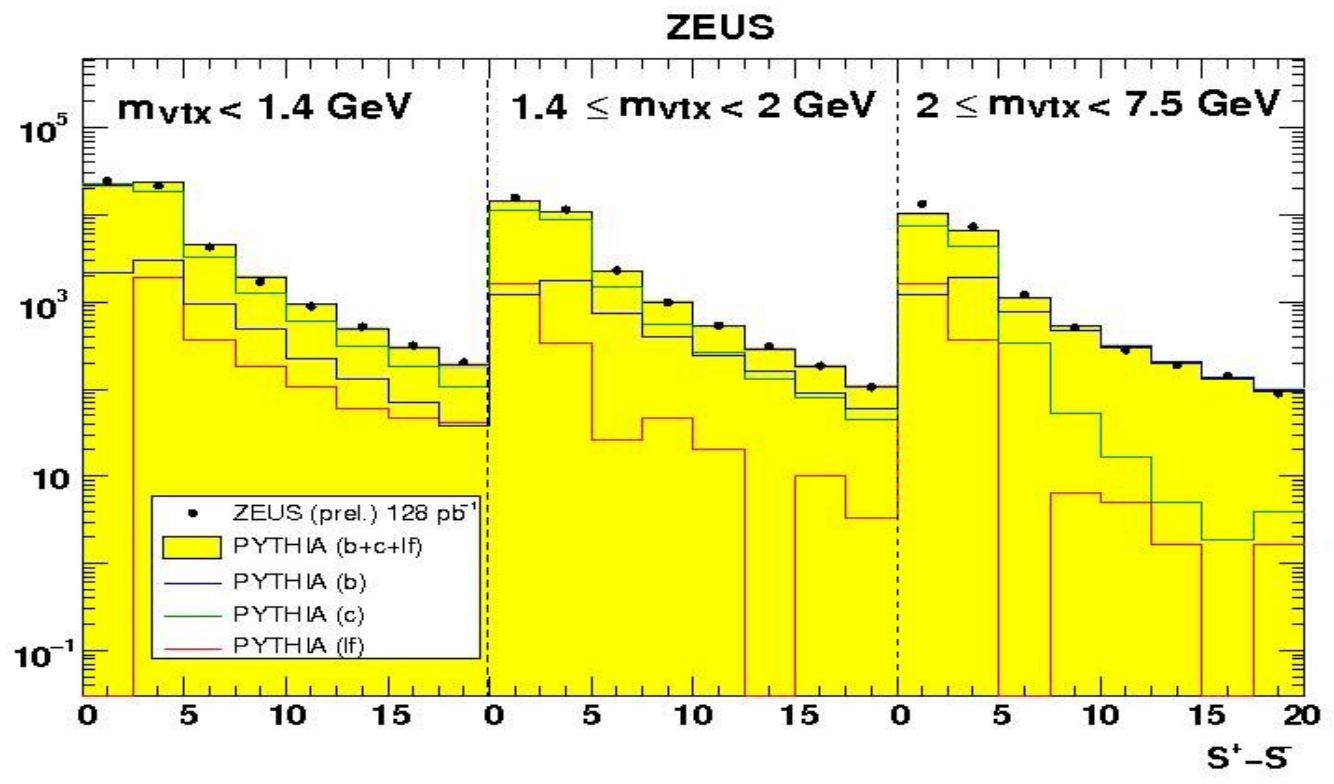




\section{Beauty PHP from inc. secondary vertexing The results}

- FMNR NLO QCD predictions:

$>\mu=\mu_{o},\left(\mu_{o} / 4\right)<\mu<\mu_{o}, m_{b}=4.75 \mathrm{GeV}, 4.5 \mathrm{GeV}<\mathrm{m}_{\mathrm{b}}<5 \mathrm{GeV}$

$>\operatorname{PDF}(\mathrm{p})=\mathrm{CTEQ} 5, \mathrm{PDF}(\gamma)=\mathrm{GRV}-\mathrm{HO}, \epsilon_{\mathrm{b}}=0.0035$

$\gg$ Hadronization corrections from PYTHIA

- Results: Good agreement with NLO QCD Good agreement with previous HERA I results (DESY-03-212) Completly different tagging technique $\rightarrow$ better precision achieved
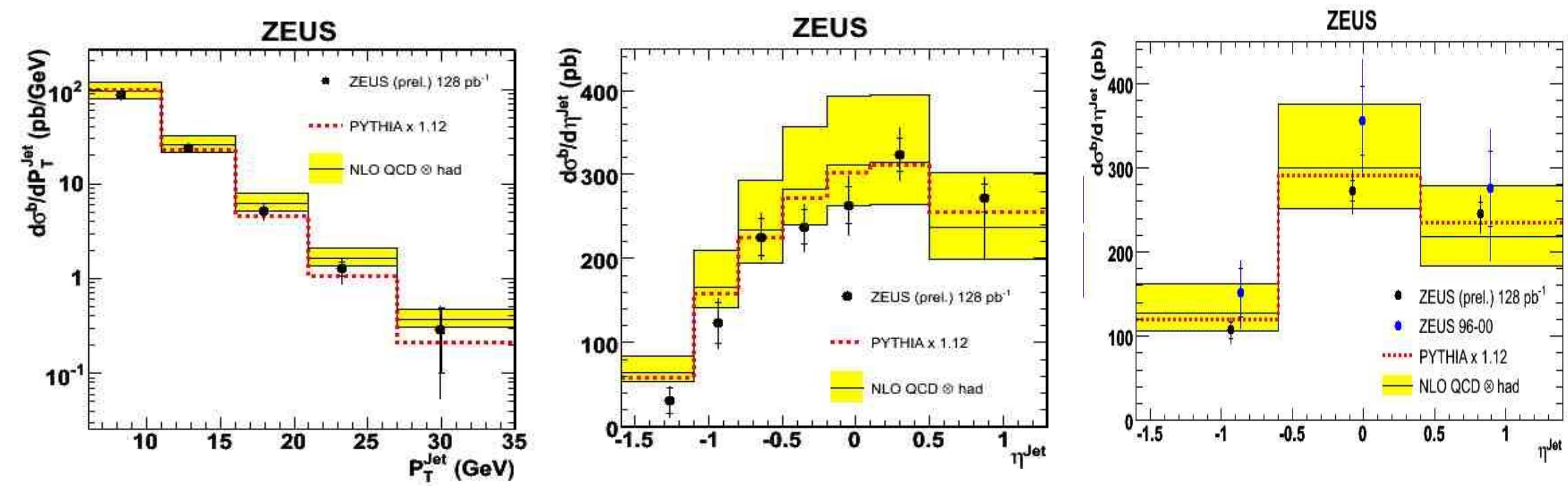


\section{Beauty PHP using decays into $\mu$ in dijet events}

\section{The experimental method}

Final result DESY-08-210 published by JHEP

Semi-leptonic decays: final state $\mu+2$ jets

Discriminant variables used :

$\gg \mathrm{P}_{\mathrm{T}}$ of muon relative to jet axis $\left(\mathrm{P}_{\mathrm{T}}{ }^{\text {rel }}\right)$

$\gg$ Muon impact parameter $(\delta)$

Combined $\mathrm{p}_{\mathrm{T}}^{\text {rel }}-\delta$ fit used

$\rightarrow$ provides complementary information
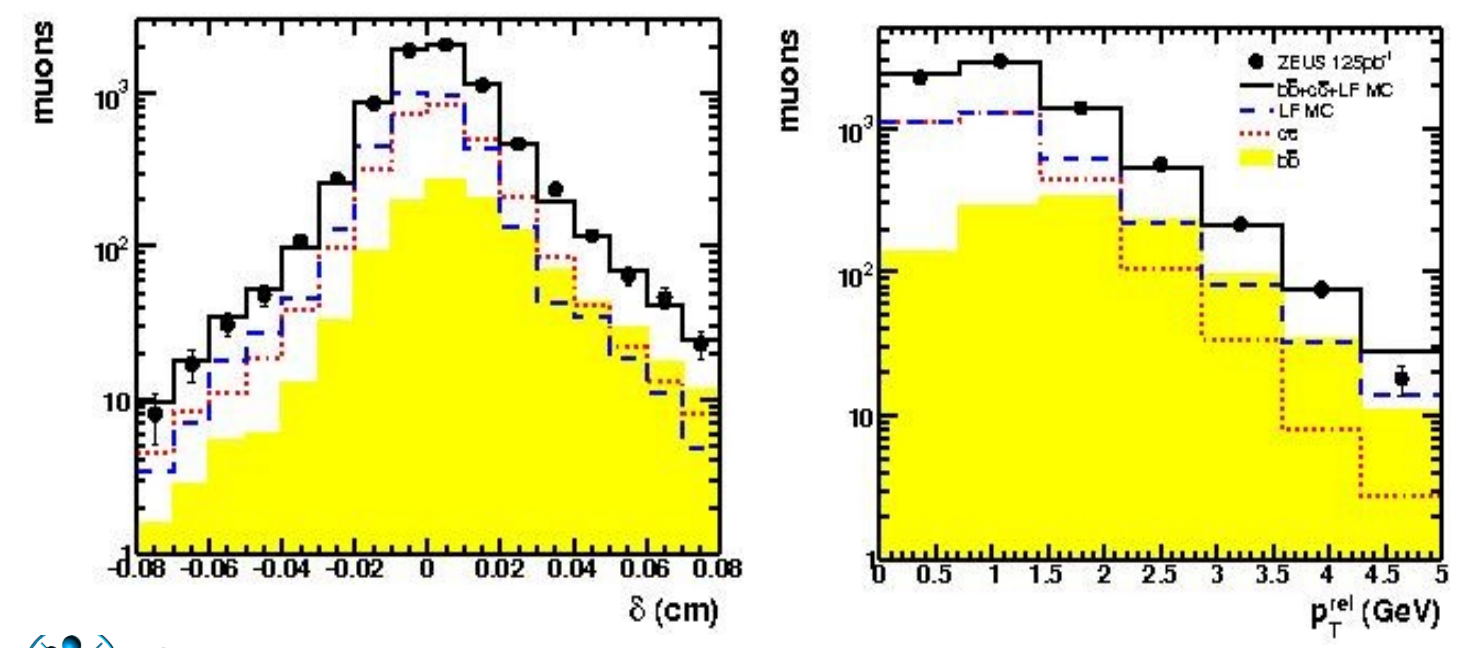

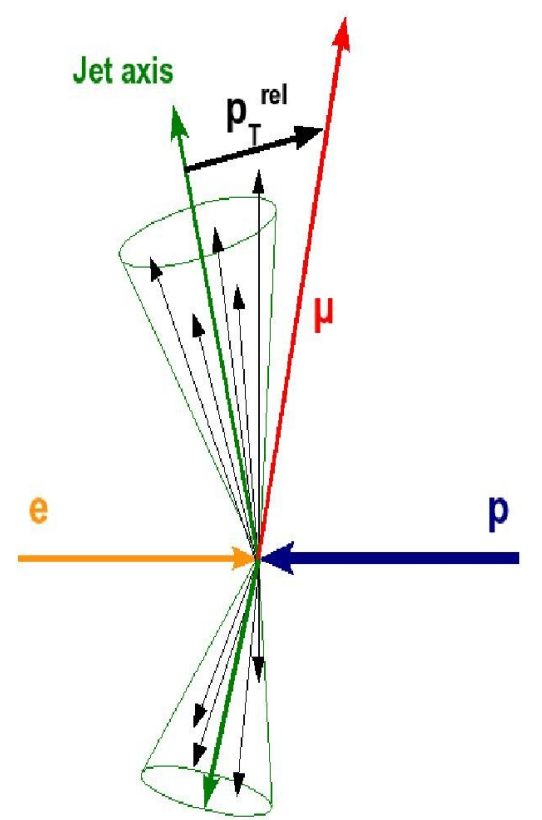

\section{The data selection}

$\mathcal{L}=125 \mathrm{pb}^{-1}$ (2005 $\mathrm{e}^{+} \mathrm{p}$ data)

- PHP selection:

veto on scattered $\mathrm{e}^{ \pm}, 0.2<\mathrm{y}_{\mathrm{JB}}<0.8$

JET selection:

2 Jets (kt-algorithm, massive)

with $\mathrm{p}_{\mathrm{T}}>7(6) \mathrm{GeV}$ and $|\eta|<2.5$

$\mu$ selection:

$\mathrm{p}_{\mathrm{T}}{ }^{\mu}>1.5 \mathrm{GeV}, 1.6<\eta<2.3$ 


\section{Beauty PHP using decays into $\mu$ in dijet events}

\section{The results}

FMNR NLO QCD predictions:

$>\mu=\mu_{o}, 0.5 \mu_{o}<\mu<2 \mu_{o}, m_{b}=4.75 \mathrm{GeV}, 4.5 \mathrm{GeV}<\mathrm{m}_{\mathrm{b}}<5 \mathrm{GeV}$

$\gg \operatorname{PDF}(\mathrm{p})=\mathrm{CTEQ} 5 \mathrm{M}, \operatorname{PDF}(\gamma)=\mathrm{GRV}-\mathrm{HO}, \epsilon_{\mathrm{b}}=0.0035$

$\gg$ Hadronization corrections from PYTHIA

Results:

$\rightarrow$ Good agreement with NLO QCD

$\rightarrow$ Good agreement with previous HERA I results $\left(\mathrm{p}_{\mathrm{T}}{ }^{\text {rel }}\right.$ only)
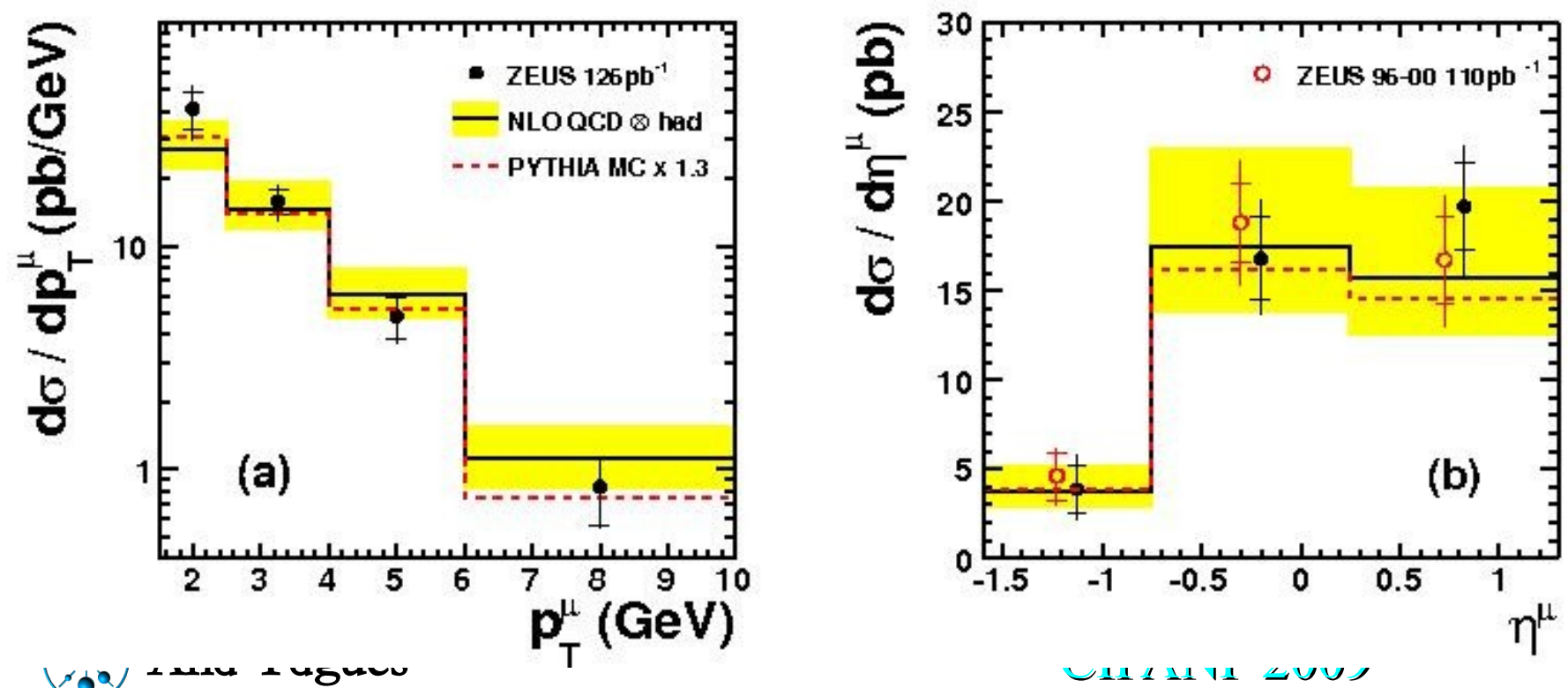

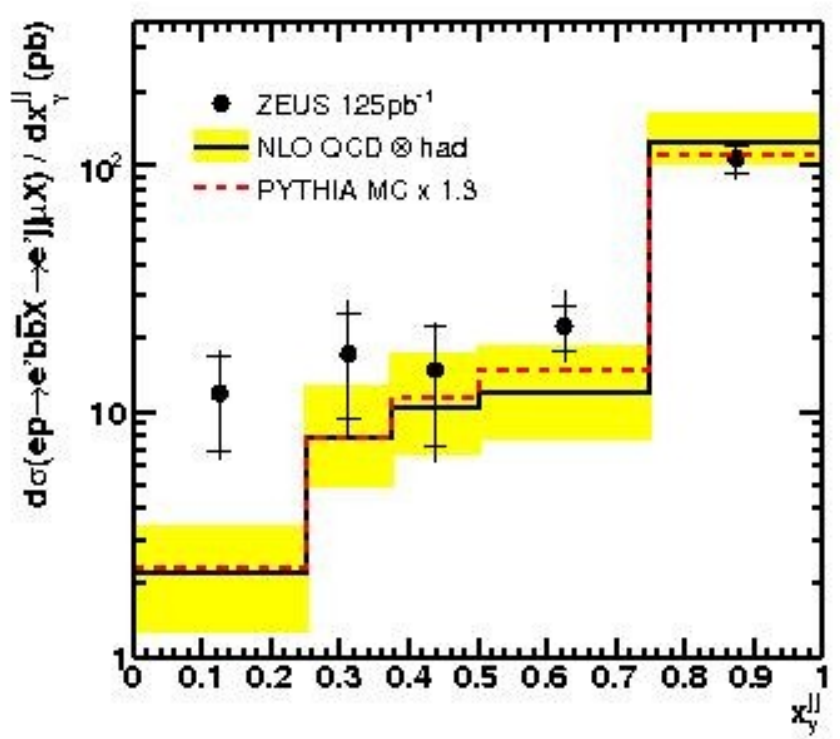

$$
\mathrm{x}_{v}^{\mathrm{ji}}=\Sigma_{\mathrm{j} 1 \mathrm{2} 2}(\mathrm{E}-\mathrm{Pz}) / \Sigma_{\mathrm{h}}(\mathrm{E}-\mathrm{Pz})
$$

at LO QCD $x_{\gamma}$ is the fraction of photon's energy entering the hard interaction

No serious discrepancy within the data and theory 
HERA

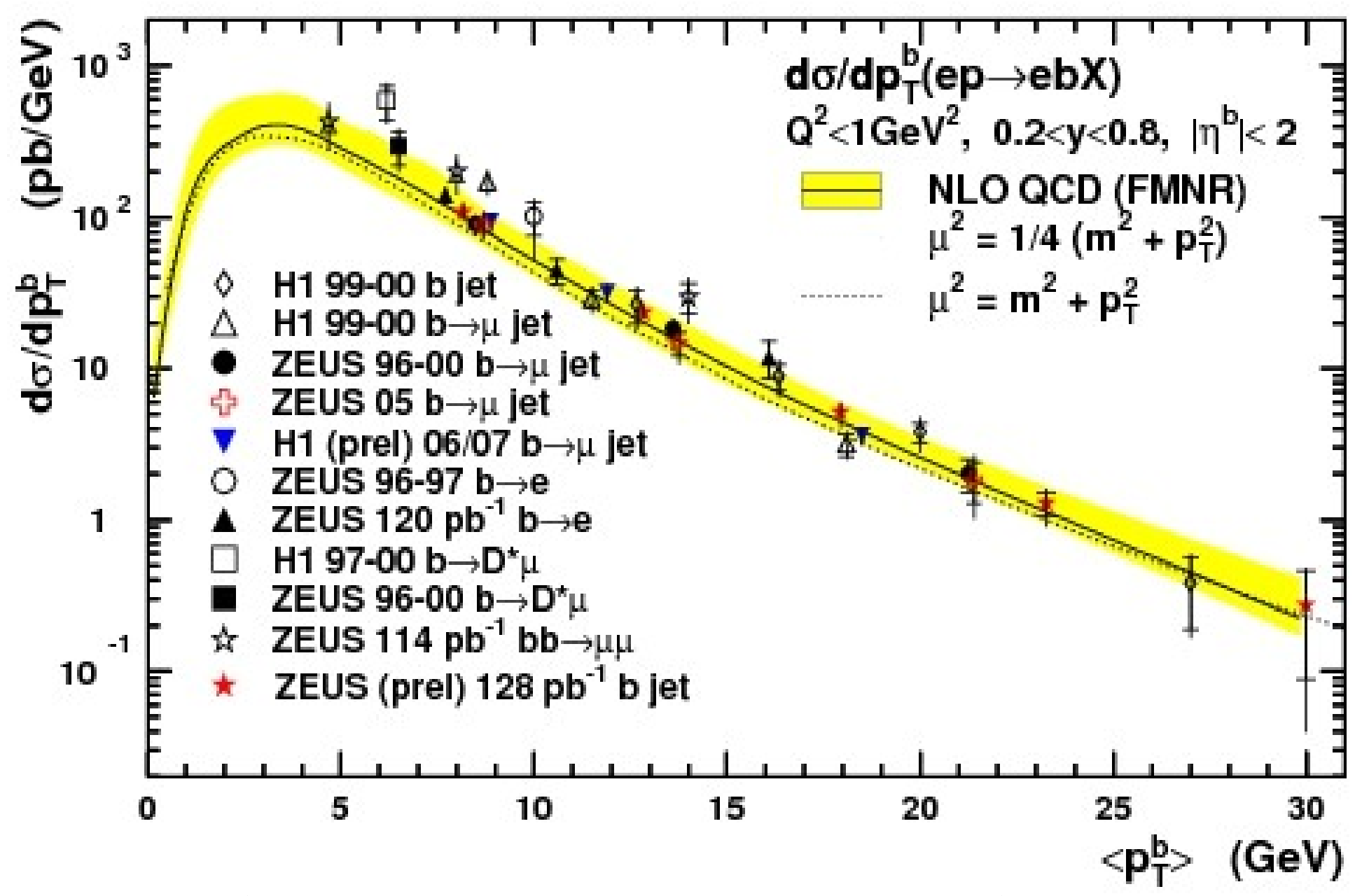

Results obtained with different methods and by different experiments. Cross sections for $b$ production extrapolated using NLO calculations. General good agreement with NLO QCD predictions 


\section{Measurements of $F_{2}^{b b}$}

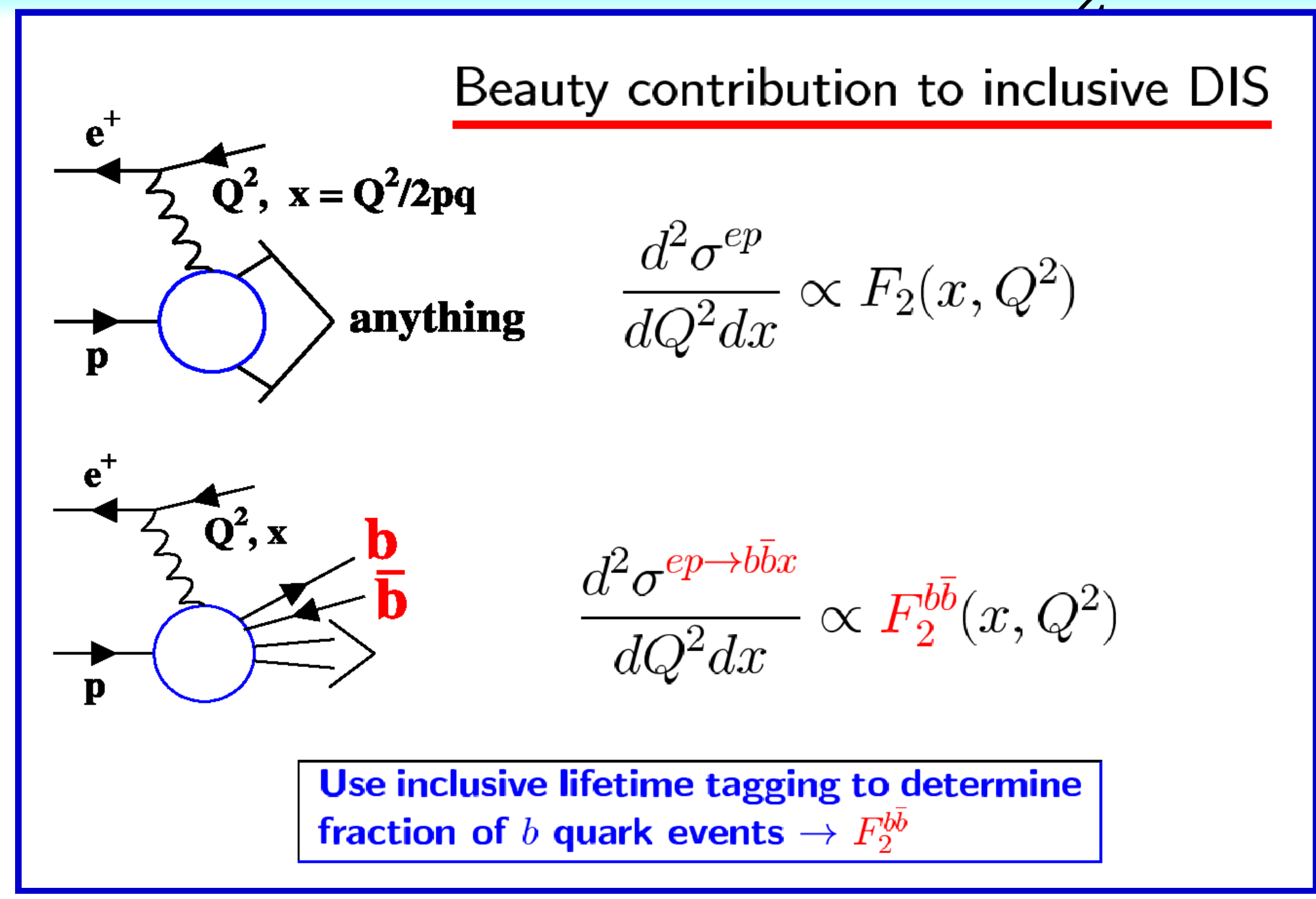

\footnotetext{
$\mathrm{H1}$, for process (ep $\rightarrow \mathrm{ebbX})$

ZEUS, for process (ep $\rightarrow$ ebbX $\rightarrow$ e $\mu \mathrm{j} X)$ 


\section{Measurement of $\mathrm{F}_{2}^{\text {bb }}$ by $\mathrm{H} 1$}

Inclusive analysis: select all tracks with hits in silicon detector and $\mathrm{p}_{\mathrm{T}}>0.3 \mathrm{GeV}$.

For each track: track significance $S=\delta / \sigma(\delta)$ with $\delta$ 三 signed impact parameter

For each reconstructed sec.vtx: significance $\mathrm{S}_{\mathrm{L}}=\mathrm{L}_{\mathrm{xy}} / \sigma\left(\mathrm{L}_{\mathrm{xy}}\right)$

For $\mathrm{N}_{\text {track }} \geqslant 3$, Neural Network $(\mathrm{NN})$ used for improved separation between $b$ and $c$

Signal extracted in each $\left(y-Q^{2}\right)$ bin using $\chi 2$-simultaneous fit to subtracted $S_{1}, S_{2}$ and NN distributions
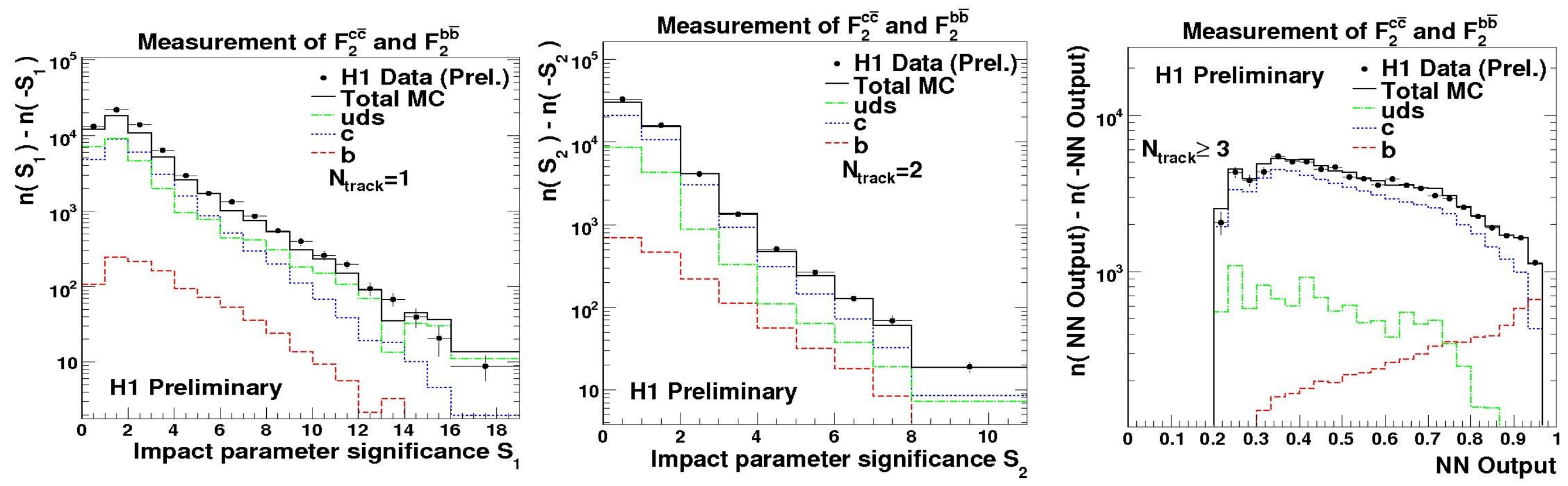


\section{Measurement of $\mathrm{F}_{2}^{\text {bb }}$ by $\mathrm{H} 1$}

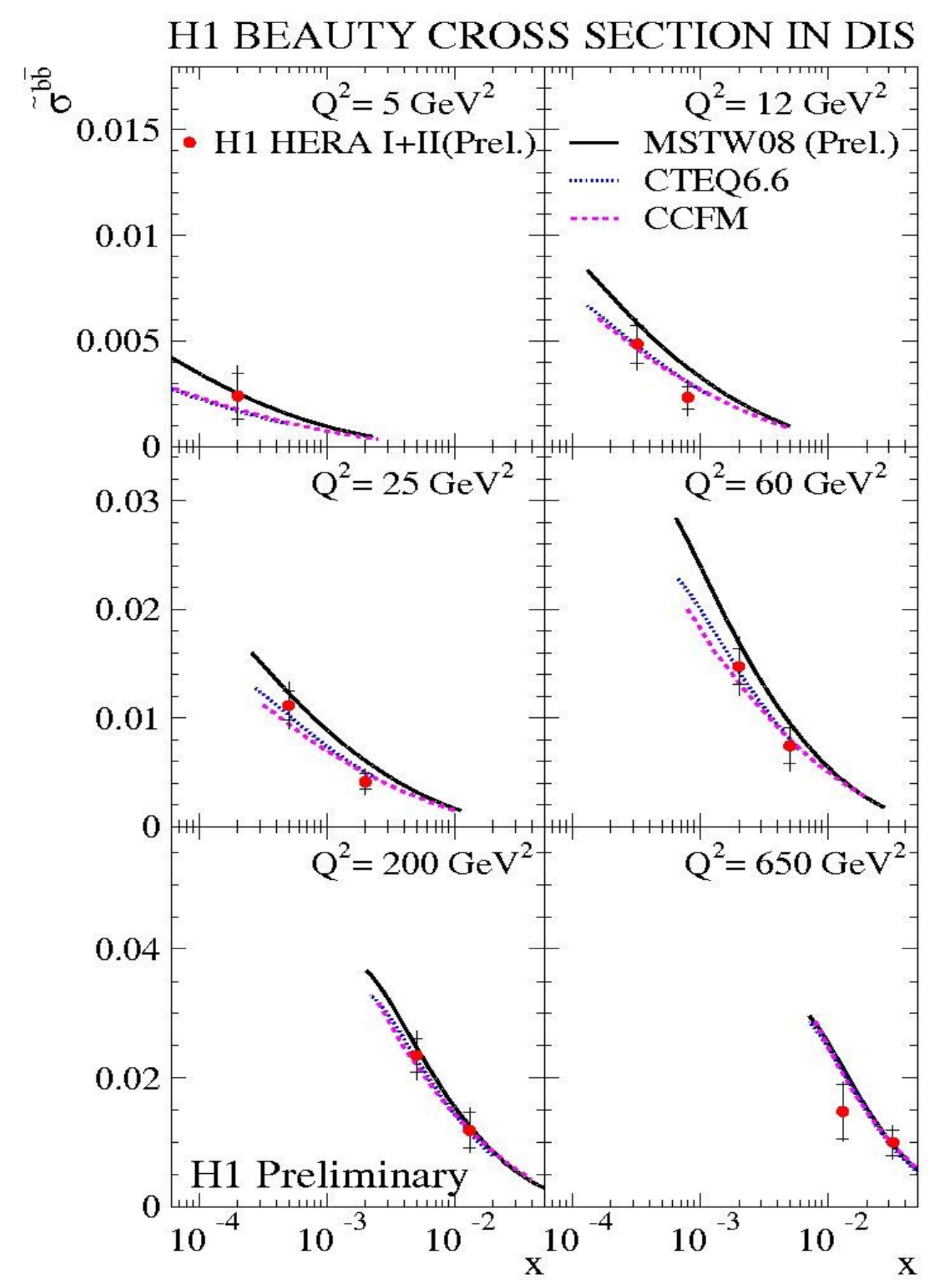

Kinematic range:

$5<\mathrm{Q}^{2}<650 \mathrm{GeV}^{2}, 0.0002 \leqslant \mathrm{x} \leqslant 0.032$

- Results compared to NLO QCD predictions and predictions based on CCFM parton evolution

Data well described by preditions

- $\mathrm{F}_{2}^{\mathrm{bb}}$ and $\mathrm{F}_{2}^{\mathrm{cc}}$ extracted simultaneously

Differences in theory are within mass and scale uncertainties (see slide 15) 


\section{Measurements of $\mathrm{F}_{2}^{\mathrm{bb}}$ by ZEUS}

Semileptonic decay into muons: $\mathrm{P}_{\mathrm{T}}{ }^{\mu}>1.5 \mathrm{GeV}$ and $-1.6<\eta^{\mu}<2.3$

Fraction of muons determined from simultaneous fit of 3 discriminating variables:

- $\mathrm{P}_{\mathrm{T}}^{\text {rel: }}: \mathrm{P}_{\mathrm{T}}$ of muon relative to associated jet axis

$\delta$ : signed impact parameter of muon

- $\mathbf{P}_{\mathrm{T}}$ miss $\mid \mu_{\text {: }}$ missing $\mathbf{P}_{\mathrm{T}}$ parallel to the muon (sensitive to $\nu$, high resolution HCAL needed)

- $\mathrm{F}_{2}^{\mathrm{bb}}\left(\mathrm{x}, \mathrm{Q}^{2}\right)=\left[\sigma_{\text {measured }}^{\mathrm{b}} / \sigma_{\mathrm{NLO}}^{\mathrm{b}}\right] \cdot \mathrm{F}_{2}^{\mathrm{bb}}{ }_{\mathrm{NLO}}\left(\mathrm{x}, \mathrm{Q}^{2}\right)$ @NLO in FFNS using HVQDIS

Kinematic range:

$\mathrm{Q}^{2}>20 \mathrm{GeV}^{2}, 0.01<\mathrm{y}<0.7$

Ana Yagües
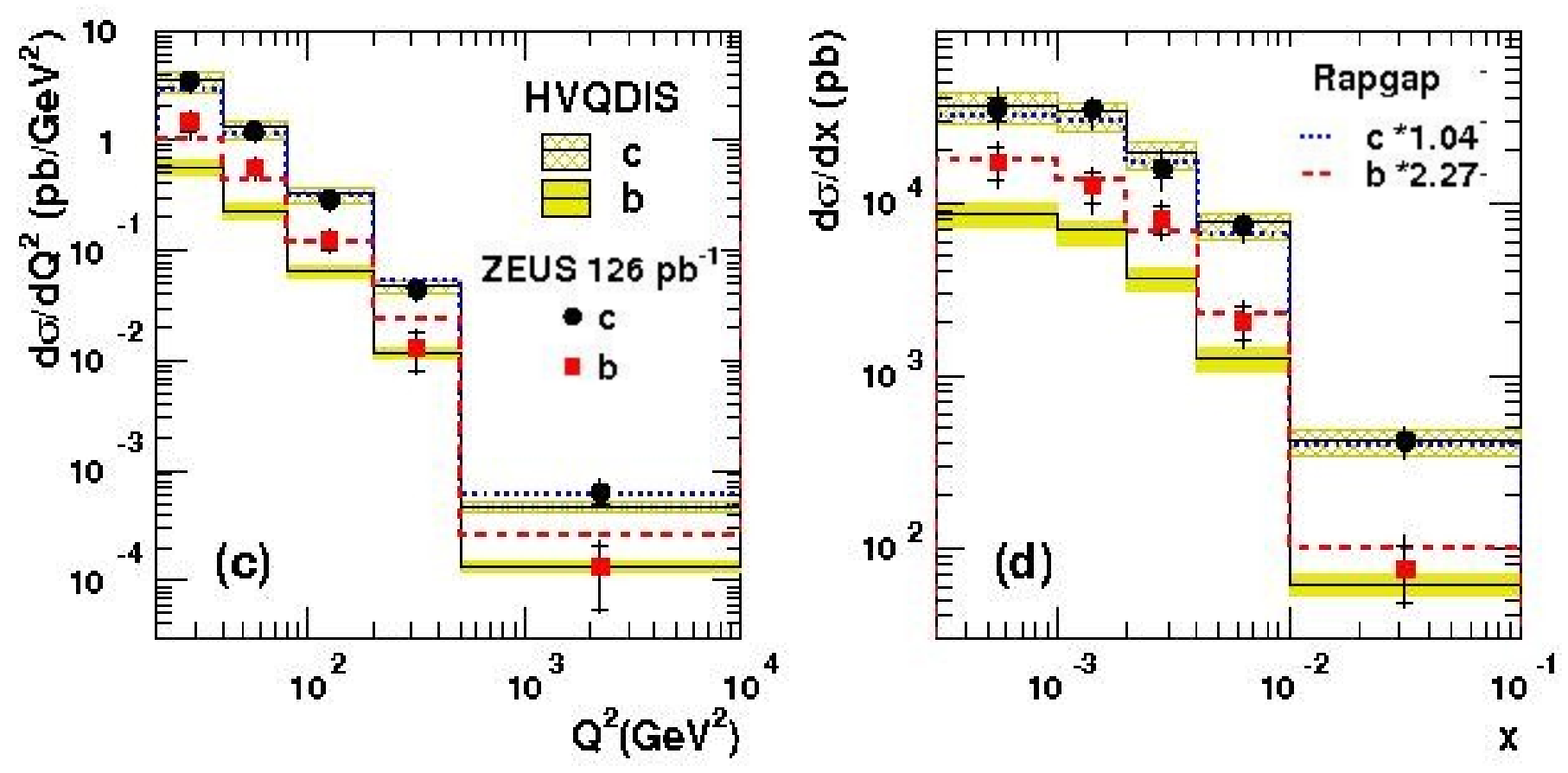


\section{Measurements of $F_{2}^{b b}$}

Latest $\mathrm{F}_{2}^{\text {bb }}$ measurements using different techniques were performed at ZEUS and $\mathrm{H} 1$

Good agreement between both coll. Good agreement between theory and data Different theory schemes agree well

Data precision can be improved by: $>$ Using the whole HERA data sample $>$ Combining H1 and ZEUS data $>$ Combining different tag methods

Ana Yagües
HERA

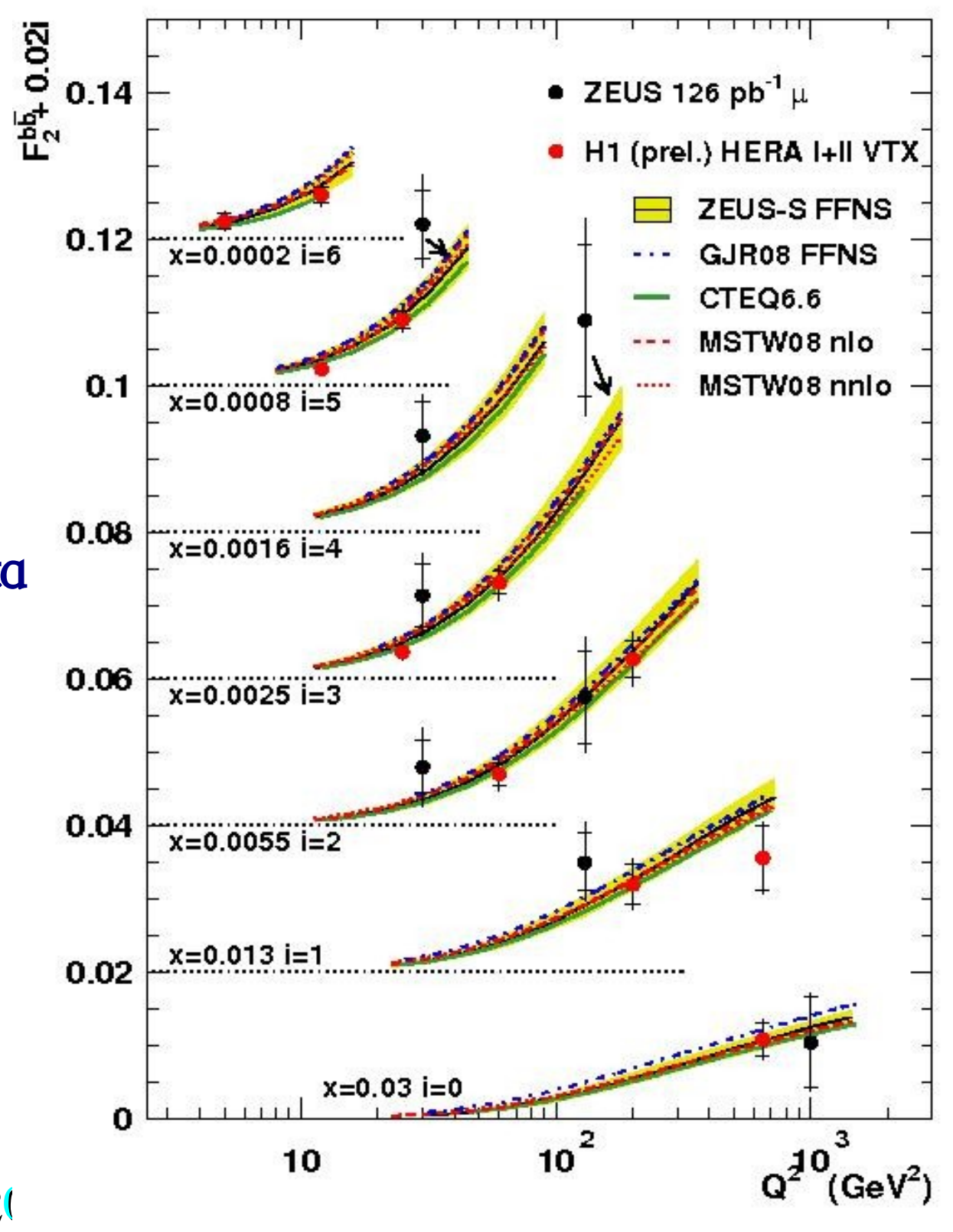




\section{Conclusions}

First ZEUS dijet inclusive PHP measurement of beauty has been presented:

$\rightarrow$ Good agreement with theory and better precision achieved than previous published result.

Different beauty measurements from ZEUS and H1 since last summer:

$\rightarrow$ Very good agreement between the results and with NLO QCD predictions

The beauty contribution to the proton structure functions has been measured at ZEUS and H1:

$\rightarrow$ Good agreement between the two collaborations, both using different methods for the analysis.

$\rightarrow$ Good agreement between theory and data. 


\section{BACK-UP SLIDES}




\section{Measurements of $F_{2}^{b b}$}

- Given the relation between double differential cross section and the proton structure functions:

$$
\mathrm{d}^{2} \sigma^{\mathrm{bb}} /\left(\mathrm{dx} \cdot \mathrm{dQ}^{2}\right)=\left(2 \pi \alpha^{2} / \mathrm{Q}^{4} \mathrm{x}\right)\left\{\left[1+(1-\mathrm{y})^{2}\right] \mathrm{F}_{2}^{\mathrm{bb}}\left(\mathrm{x}, \mathrm{Q}^{2}\right)-\mathrm{y}^{2} \mathrm{~F}_{\mathrm{L}}^{\mathrm{bb}}\left(\mathrm{x}, \mathrm{Q}^{2}\right)\right)
$$

$\rightarrow$ The reduced b cross section can be defined from the double as

$$
\tilde{\sigma}^{\mathrm{bb}}\left(\mathrm{x}, \mathrm{Q}^{2}\right)=\left[\mathrm{d}^{2} \sigma^{\mathrm{bb}} /\left(\mathrm{dx} \cdot \mathrm{dQ} \mathrm{Q}^{2}\right)\right]\left[\mathrm{xQ} \mathrm{Q}^{4} /\left(2 \pi \alpha^{2}\left(1+(1-\mathrm{y})^{2}\right)\right]\right.
$$

- $\mathrm{F}_{2}{ }^{\mathrm{bb}}$ can be calculated from the ratio of measured and theory cross sections:

$$
\mathrm{F}_{2}^{\mathrm{bb}}\left(\mathrm{x}, \mathrm{Q}^{2}\right)=\left[\sigma_{\text {measured }} / \sigma_{\mathrm{NLO}}\right] \cdot \mathrm{F}_{2}^{\mathrm{bb}}{ }_{\mathrm{NLO}}\left(\mathrm{x}, \mathrm{Q}^{2}\right)
$$

\section{H1, for process $(\mathrm{ep} \rightarrow \mathrm{ebbX})$}

\section{ZEUS, for process $(\mathrm{ep} \rightarrow \mathrm{ebbX} \rightarrow \mathrm{e \mu j} \mathrm{X})$}




\section{Beauty PHP from inc. secondary vertexing}

Control Distributions
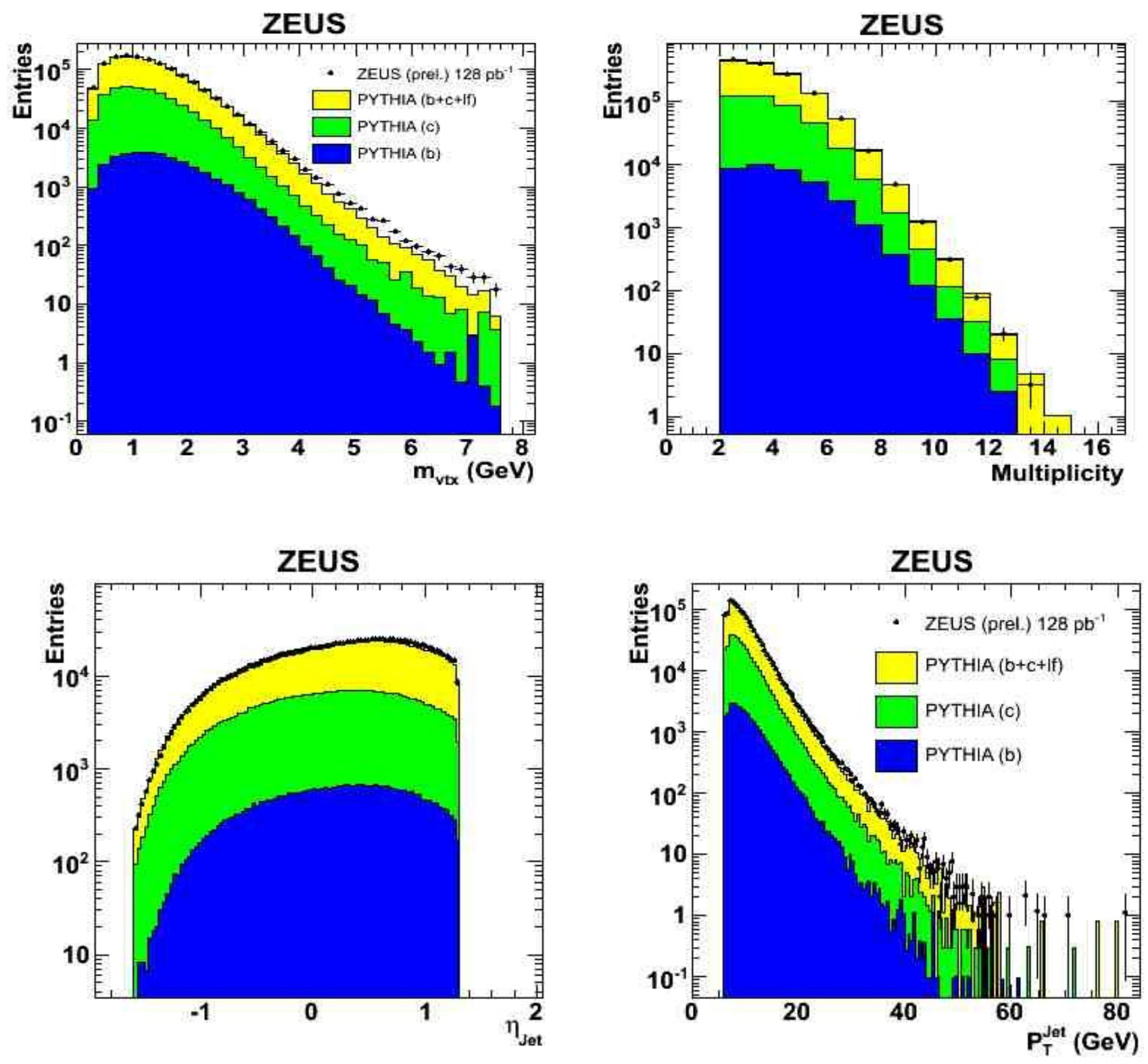


\section{Beauty PHP using decays into $\mu$ in dijet events}
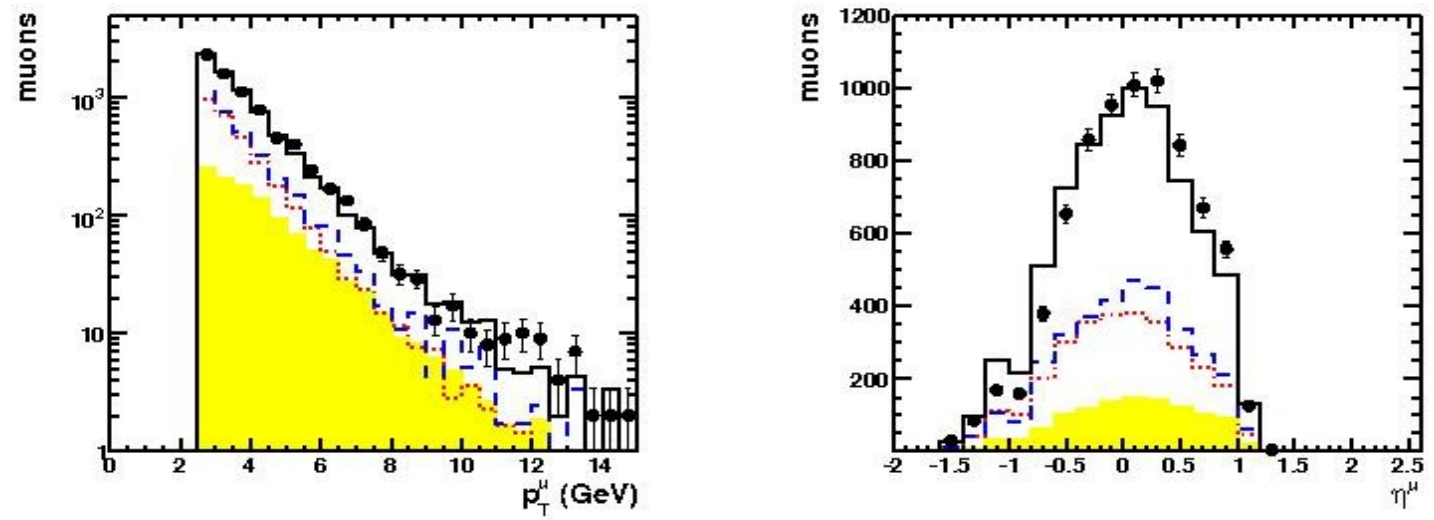

Control Distributions
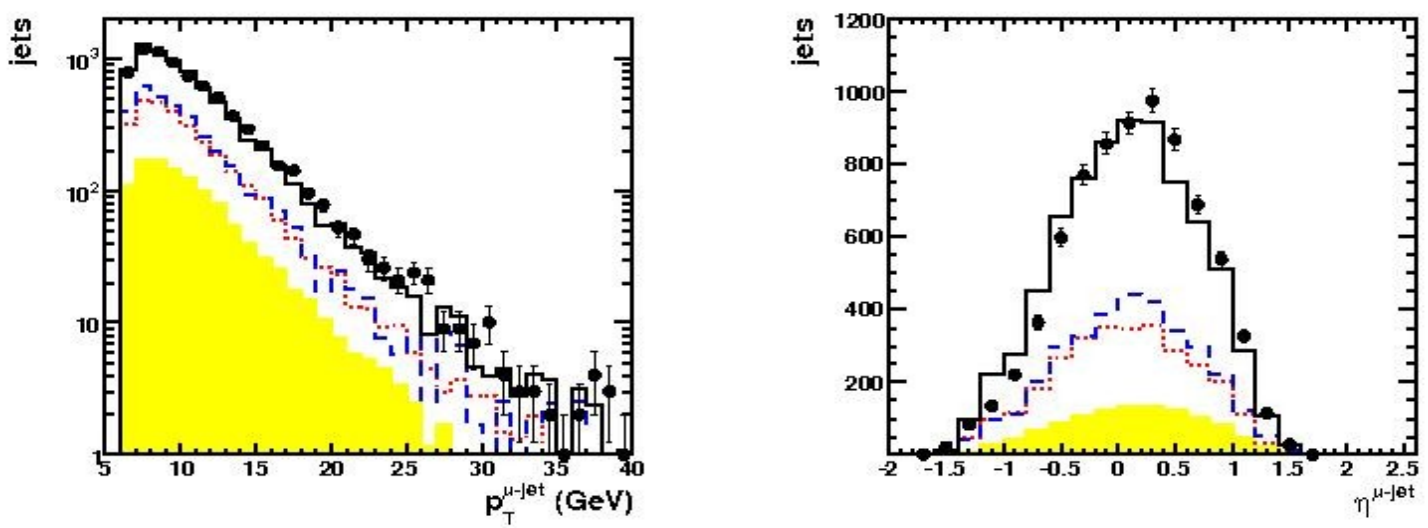

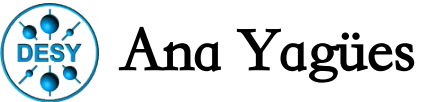

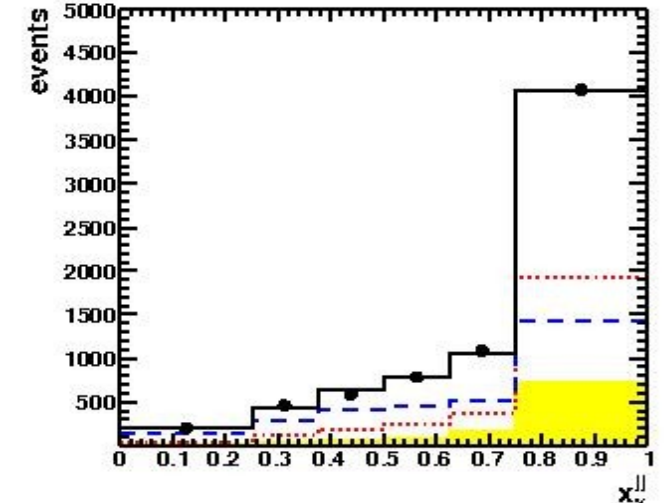

- ZEUS $125 \mathrm{pb}^{-1}$

$-b \bar{b}+c \bar{c}+L F M C$ - - LF MC

…… $c \overline{\mathrm{C}}$ $\mathrm{b} \overline{\mathrm{b}}$ 


\section{Beauty PHP using decays into $\mu$ in dijet events}

\section{The experumental methoa}

Final result DESY-08-210 published by JHEP

Semi-leptonic decays: final state $\mu+2$ jets

Discriminant variables used :

$\gg \mathrm{P}_{\mathrm{T}}$ of muon relative to jet axis $\left(\mathrm{P}_{\mathrm{T}}\right.$ rel)

$\gg$ Muon impact parameter ( $\delta$ )

Combined $\mathrm{p}_{\mathrm{T}}{ }^{\text {rel }}-\delta$ fit used

$\rightarrow$ provides complementary information
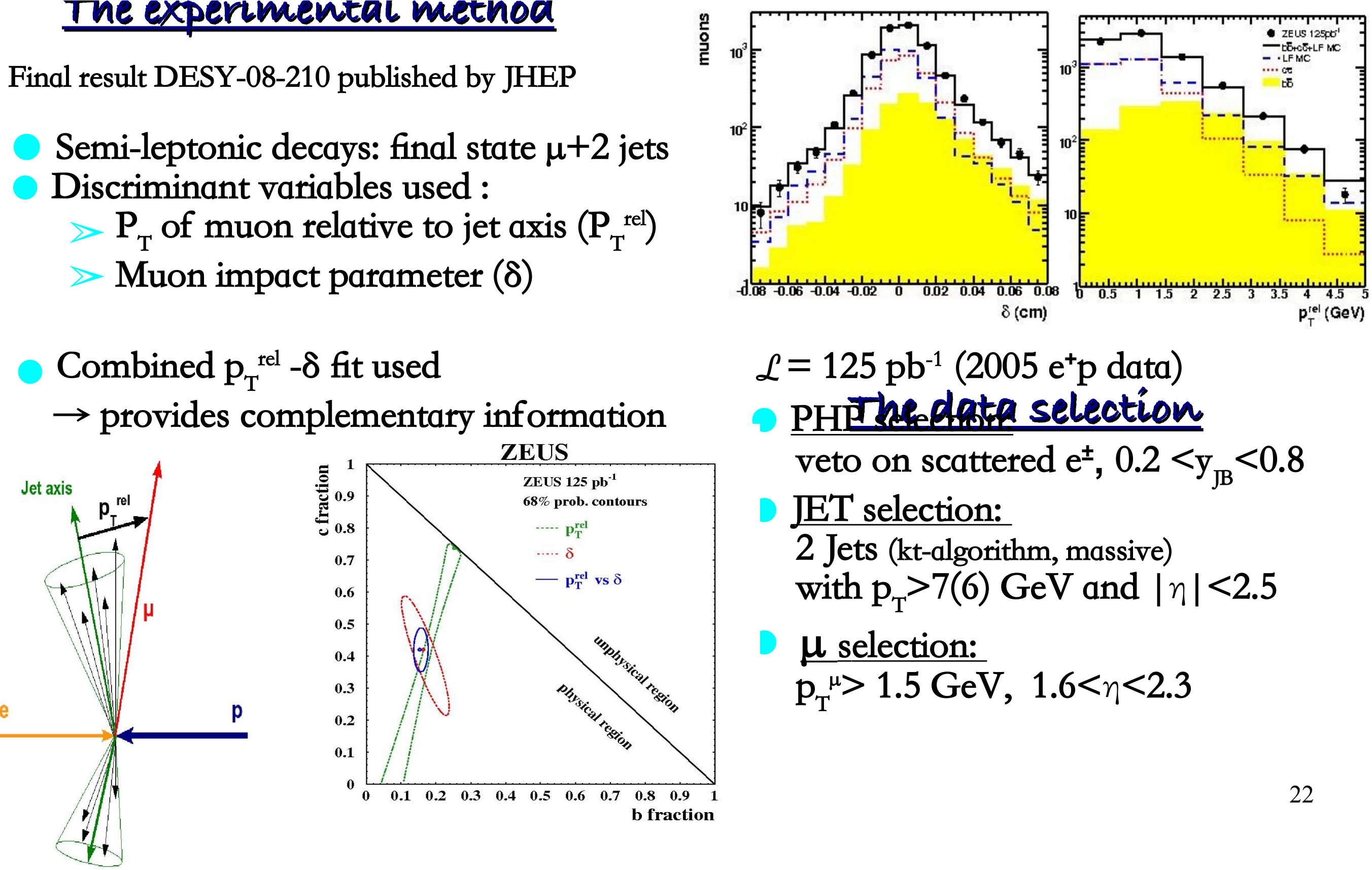

\section{ZEUS}

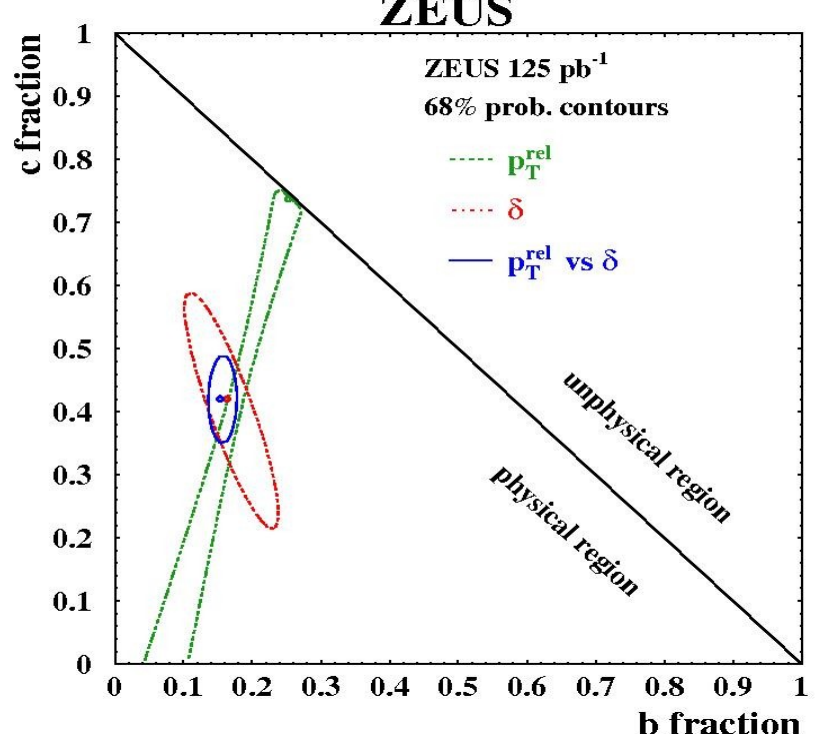

$\mathcal{L}=125 \mathrm{pb}^{-1}\left(2005 \mathrm{e}^{+} \mathrm{p}\right.$ data $)$

- PHThedlata selection veto on scattered $\mathrm{e}^{ \pm}, 0.2<\mathrm{y}_{\mathrm{JB}}<0.8$

JET selection:

2 Jets (kt-algorithm, massive) with $\mathrm{p}_{\mathrm{T}}>7(6) \mathrm{GeV}$ and $|\eta|<2.5$

$\mu$ selection: $\mathrm{p}_{\mathrm{T}}{ }^{\mu}>1.5 \mathrm{GeV}, 1.6<\eta<2.3$ 


\section{List of results since last summer}

$\checkmark$ Latest beauty results at HERA:

PHP events with 2 jets + sec.vtx (ZEUS-prel-og-005)

PHP events with 2 jets $+1 \mu$ (DESY-o8-210, by ZEUS Coll.)

$\left\{\begin{array}{l}\text { DIS decays into } \mu \text { 's (DESY-o9-56 by ZEUS Coll.) } \\ \text { DIS events using sec.vtx (H1-prel-o8-173 by H1 Coll.) }\end{array}\right.$

PHP events with 2 jets $+1 \mu$ (H1-prel-o8-o71, by H1 Coll.)

- PHP events with 2 jets +1 e (PRD 78,072001 (2008), by ZEUS Coll.)

- Events with $1 D^{*}+1 \mu$ (EPJ C50 (2007) 299-314,by ZEUS Coll.)

- Events with $2 \mu$ 's (JHEPo2 (2009) 032, by ZEUS Coll.) 